\title{
Control analgésico continuo en paciente con múltiples fracturas costales mediante colocación de catéter en el plano profundo del músculo erector de la espina
}

\author{
Continuous analgesic control in a patient with multiple \\ rib fractures by placing a catheter in the deep plane \\ of the erector muscle of the spine
}

A. M. Gasalla Cadórniga, E. Domíngez Suárez¹, J. M. López González¹, B. M. Jiménez Gómez¹ y G. Illodo Miramontes ${ }^{2}$

${ }^{1}$ Servicio de Anestesiología y Reanimación. Hospital Universitario Lucus Augusti. Lugo, España.

¿2Servicio de Anestesiología y Reanimación. Complejo Hospitalario Universitario de Vigo, España

\section{ABSTRACT}

Rib fractures have a high prevalence in our society.

An adequate and precocious analgesic control is essential to avoid respiratory complications and favor a rapid functional recovery in patients with rib fractures.

Different strategies have been used for analgesic control, from intravenous drugs to more classic regional techniques such as epidural catheter or thoracic paravertebral block.

Ultrasound-guided blockade of the deep plane of the erector spine muscle (ESPB) is an effective alternative in the management of acute pain derived from rib fractures, allowing physiotherapy and early rehabilitation.

Key words: Rib fracturas, block the deep plane of the erector muscle of the spine, neural blocks intervencionism ultrasound, patient control analgesia.

\section{RESUMEN}

Las fracturas costales tienen una elevada prevalencia en nuestra sociedad.

Un adecuado y precoz control analgésico resulta fundamental a la hora de evitar complicaciones respiratorias y favorecer una rápida recuperación funcional en los pacientes con fracturas costales.

Se han empleado diferentes estrategias para dicho control analgésico, desde fármacos intravenosos hasta técnicas regionales más clásicas como el catéter epidural o el bloqueo paravertebral torácico.

El bloqueo ecoguiado del plano profundo del músculo erector de la espina (ESPB) constituye una alternativa eficaz en el manejo del dolor agudo derivado de las fracturas costales, permitiendo una fisioterapia y rehabilitación precoz.

Palabras clave: Fracturas costales, bloqueo del plano profundo del músculo erector de la espina, bloqueos neuronales, ecografía intervencionista, analgesia controlada por el paciente.
Gasalla AM, Domínguez Suárez E, López González JM, Jiménez Gómez BM, Illodo Miramontes G. Control analgésico continuo en paciente con múltiples fracturas costales mediante colocación de catéter en el plano profundo del músculo erector de la espina. Rev Soc Esp Dolor. 2020;27(4):269-272
Recibido: 10-03-2020

Aceptado: 06-05-2020

Correspondencia: Álvaro Manuel Gasalla Cadórniga alvarogasalla@hotmail.com 


\section{INTRODUCCIÓN}

Las fracturas costales tienen una elevada prevalencia en nuestra sociedad, sobre todo en relación con accidentes de tráfico y otro tipo de politraumatismos (accidentes laborales, domésticos, etc.).

Un adecuado y precoz control analgésico resulta fundamental a la hora de evitar complicaciones respiratorias y favorecer una rápida recuperación funcional.

Se han empleado diferentes estrategias para dicho control analgésico, desde fármacos intravenosos, hasta técnicas regionales más clásicas como el catéter epidural o el bloqueo paravertebral.

El bloqueo ecoguiado del plano profundo del músculo erector de la espina (ESPB), cuya diana son los ramos ventral y dorsal de los nervios raquídeos torácicos, aparece como una alternativa eficaz y segura en la estrategia analgésica de estos pacientes [1].

\section{CASO CLÍNICO}

Presentamos el caso de un varón de 48 años, sin antecedentes personales de interés, que acudió al Servicio de Urgencias tras caída casual por las escaleras de su domicilio. Refería un dolor de gran intensidad en la pared costal izquierda, con una puntuación en la escala verbal numérica (EVN) de 9/10.

A la exploración física presentaba unas cifras de tensión arterial 150/90 mmHg, saturación arterial de $\mathrm{O}_{2}$ de $96 \%$ al aire ambiente, murmullo vesicular conservado, no focalidad neurológica y dolor importante a la palpación de la pared costal izquierda. En los estudios de imagen realizados se evidenciaron múltiples fracturas costales en el hemitórax izquierdo, desde la $6 .^{a}$ a la 9. ${ }^{a}$ costillas, sin evidencia de afectación pleuropulmonar aguda (Figura 1).

Tras esta exploración, se decidió ingreso en la unidad de observación y tratamiento analgésico conservador, iniciándose tratamiento intravenoso con AINE y derivados opioides. Se avisó al Servicio de Anestesia para valoración y optimización del control analgésico por mal control del dolor a pesar de la analgesia instaurada.

Se le realizó un bloqueo ecoguiado ESPB con colocación de catéter para analgesia continua posterior. Para ello, se colocó al paciente en posición de decúbito lateral derecho, y se procedió a la preparación de la zona de punción con povidona yodada, colocación de campo estéril y elección de sonda ecográfica lineal de alta frecuencia. Se identificó la apófisis espinosa de la 6. ${ }^{a}$ vértebra torácica y a una distancia de $3 \mathrm{~cm}$ lateral se identificó el proceso transverso de la misma. En la imagen ecográfica, visualizamos el plano subcutáneo y varios grupos musculares que de superficial a profundo corresponden con el músculo trapecio, romboides y erector de la columna, todos ellos sobre la apófisis transversa vertebral (Figura 2).

Se introdujo una aguja 25 G, sistema E-Cath Pajunk, en sentido craneocaudal, depositando el anestésico local $(A L)$ en el plano fascial profundo del músculo erector de la columna, observando en la imagen ecográfica la difusión del mismo a nivel subfascial, tanto en sentido craneal como caudal, así como la elevación de los grupos musculares. En este caso se empleó un

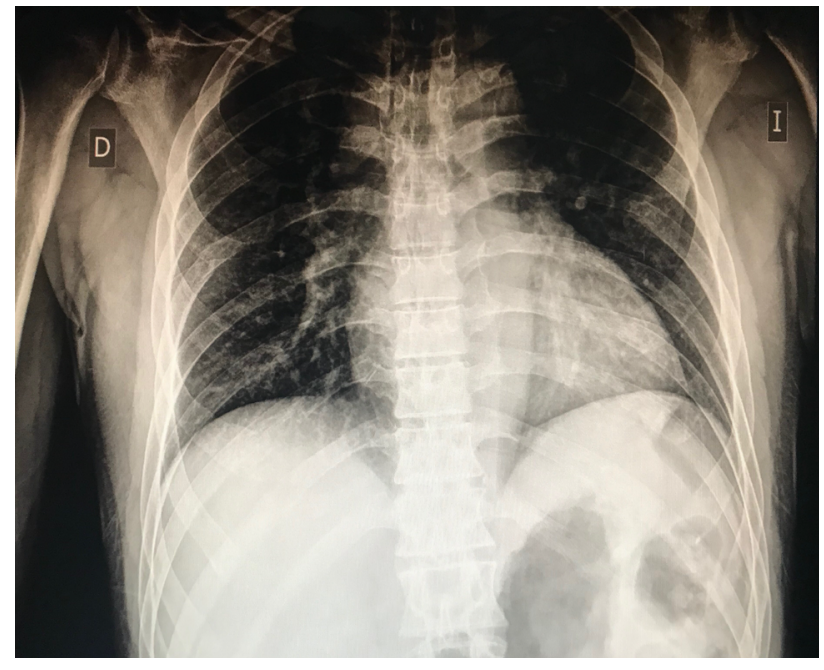

Fig. 1. Fracturas costales en la radiografía de tórax.

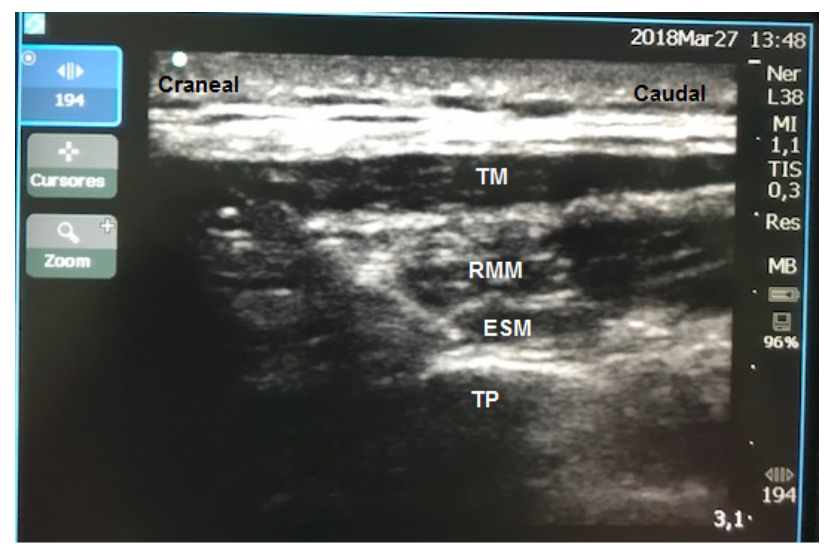

Fig. 2. Imagen ecográfica y planos musculares.

volumen de $15 \mathrm{ml}$ de levobupivacaína al 0,25 \% para, posteriormente, introducir un catéter uniperforado en dicho plano fascial. Una vez confirmada la correcta colocación del catéter con una nueva administración de $5 \mathrm{ml}$ del mismo $A L$, se procedió a su fijación en piel y protección (Figura 3).

A los 10 minutos de la técnica, el paciente refirió disminución de la intensidad del dolor (EVN 5/10). A los 20 minutos, refirió un dolor con puntuación de EVN de 3-4/10. A la exploración sensitiva, el paciente presentaba un nivel sensitivo al estímulo frío que alcanzaba hasta nivel dermatomérico T10.

Se dejó pautada una bomba de infusión continua tipo PCA (Analgesia Controlada por el Paciente) con una solución de levobupivacaína al 0,125\%, a un ritmo de infusión de $5 \mathrm{ml} / \mathrm{h}$ y bolos de rescate de $5 \mathrm{ml}$ cada 60 min, además de paracetamol y dexketoprofeno intravenosos (i.v.) alternos cada 8 horas a demanda del paciente. 


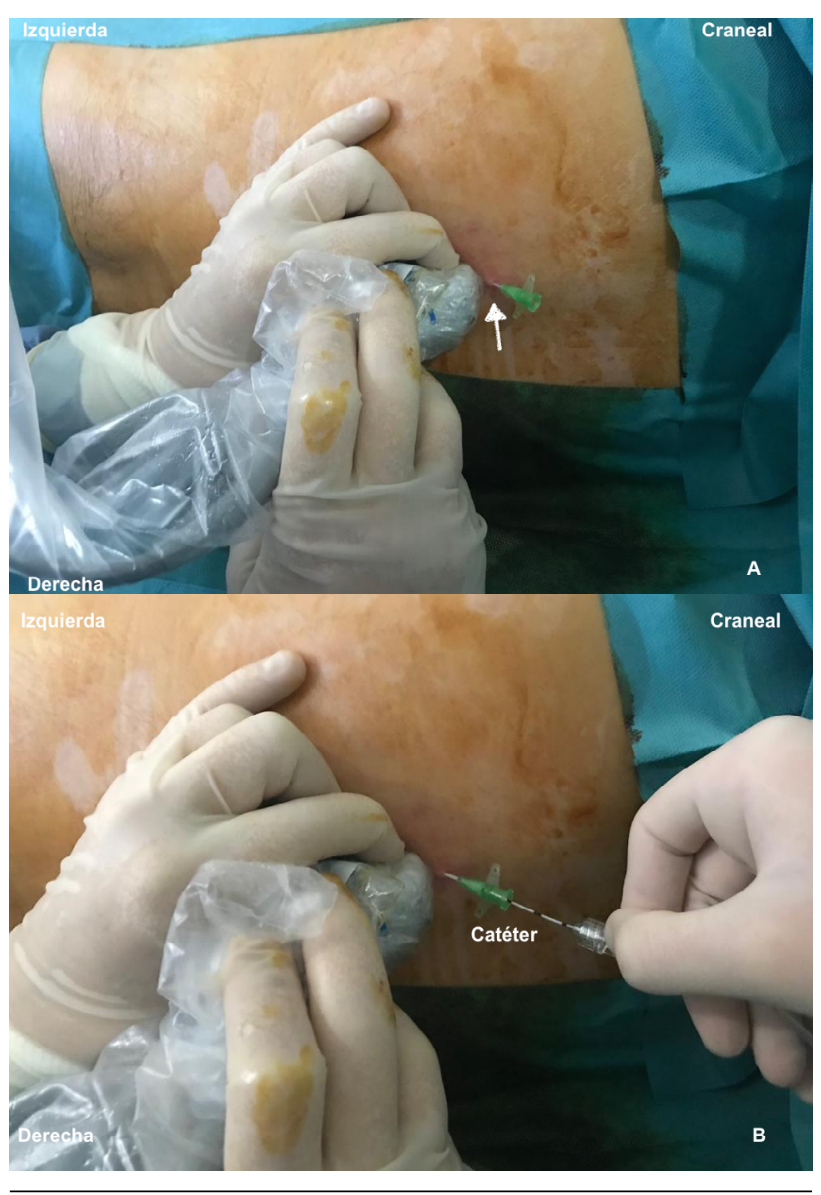

Fig. 3. Punción e inserción del catéter.

En las primeras 24 horas, el paciente precisó tres bolos de rescate administrados por el catéter, así como la administración de paracetamol pautado, refiriendo EVN < 3 en reposo, que aumentaba a un EVN 5/10 con las movilizaciones y al inicio de la fisioterapia respiratoria.

A las 48 horas, se mantenía la bomba de infusión PCA con un ritmo de infusión de $5 \mathrm{ml} / \mathrm{h}$, sin que el paciente hubiese precisado ningún bolo de rescate por el catéter, pudiendo reducir la analgesia i.v. de manera progresiva, con buena tolerancia a la rehabilitación y fisioterapia respiratoria [EVN $<3$ en reposo y $<5$ con el movimiento).

A las 72 horas, se descendió progresivamente el ritmo de infusión de la bomba de infusión PCA hasta su retirada al $6 .^{\circ}$ día de ingreso, con buena tolerancia al paso de la medicación endovenosa a la vía oral.

Al 7. ${ }^{\circ}$ día fue dado de alta a domicilio.

\section{DISCUSIÓN}

Las fracturas costales son relativamente frecuentes y extremadamente dolorosas. Un manejo precoz e intensivo del dolor previene futuras complicaciones respiratorias, además de favorecer una recuperación funcional más temprana [2,3-5].

En el contexto de este manejo analgésico surgen nuevas alternativas a los tratamientos con dosis elevadas de opioides y a las técnicas clásicas de anestesia regional neuroaxial [epidural y paravertebral], como son las técnicas de bloqueos fasciales de la pared torácica (ESPB, retrolaminar, etc.), las cuales tienen una curva de aprendizaje sencilla y en pacientes politraumatizados suponen una alternativa eficaz con menor riesgo de complicaciones con respeto a las técnicas clásicas (4).

La técnica de bloqueo fascial empleada en nuestro caso clínico, el ESPB con colocación de catéter, parece tener una buena eficacia analgésica, con la ventaja de su seguridad y su sencilla realización, disminuyendo la aparición de los riesgos asociados a las técnicas más clásicas, tales como neumotórax, hipotensión o daño neuronal, con resultados similares. Favorece la fisioterapia respiratoria, así Adhikary y cols. demostraron un incremento de los volúmenes inspiratorios medidos por espirometría en pacientes con fracturas costales tras la realización de esta técnica $(6,7)$. Además puede constituir una alternativa analgésica en determinados pacientes a tratamiento antiagregante o anticoagulante, valorando los riesgos/beneficios [8].

El objetivo del ESPB es conseguir la difusión del anestésico local $(A L)$ hasta alcanzar el ramo ventral y dorsal de los nervios espinales, para así asegurar una correcta analgesia de la pared torácica desde la parte más posterior a la anterior, así como analgesia visceral y somática en algunos casos. También se ha descrito la difusión del AL en sentido craneocaudal $(9,10)$. Como en la mayoría de los bloqueos fasciales, el ESPB se basa en depositar altos volúmenes de $\mathrm{AL}$ a bajas concentraciones del mismo para obtener una analgesia eficaz. De Cassai y cols. estimaron un volumen de $3,4 \mathrm{ml}$ de $A L$ para cada nivel vertebral deseado alcanzar $(11,12)$. En otro estudio de Luftig y cols. analizaron específicamente el volumen y la concentración empleados en 49 casos de ESPB para analgesia por fracturas costales con el objetivo de encontrar unos protocolos óptimos, creando una guía basada en el peso. Sin embargo, no existe un claro consenso al respecto sobre la dosificación y la concentración exacta del AL [13]. Probablemente, estas puedan variar según el objetivo analgésico que se pretenda conseguir.

Una de las potenciales ventajas de este bloqueo es la posibilidad de colocación de un catéter en ese plano fascial y poder prolongar la analgesia del paciente. La técnica de colocación del mismo suele ser sencilla y facilmente reproducible (10). Es importante destacar que hay que prestar especial atención a la comprobación, fijación y cuidado del catéter con el fin de evitar su desplazamiento a otro plano fascial o salida accidental del mismo, con la consiguiente pérdida de eficacia analgésica $(14,15)$.

Quizá el mayor riesgo asociado al ESPB radique en la toxicidad sistémica por anestésico local empleado, la cual se puede evitar con una correcta dosificación [16]. Entre las limitaciones de esta técnica podemos mencionar la necesidad de un conocimiento básico del 
operador con la ultrasonografía y la sonoanatomía, la colocación del paciente en unidades de urgencias/cuidados críticos y la posible variabilidad interindividual en la difusión del anestésico local [16].

\section{CONCLUSIÓN}

El bloqueo ESPB constituye una alternativa eficaz en el manejo del dolor derivado de las fracturas costales múltiples, así como un elemento importante a tener en cuenta en las estrategias analgésicas multimodales para múltiples procedimientos e intervenciones. Permite un rápido y efectivo control del dolor, así como una fisioterapia y rehabilitación precoz, disminuyendo los riesgos y efectos adversos de otras técnicas clásicas.

\section{BIBLIOGRAFÍA}

1. Forero M, Adhikary SD, López H, Tsui C, Chin KJ. The erector spinae plane block: A novel analgesic technique in thoracic neuropatic pain. Reg Anesth Pain Med. 2016;41(5):621-7. DOI: 10.1097/AAP.0000000000000451.

2. Luftig J, Mantuani D, Herring AA, Dixon B, Clattenburg E, Nagdev A. Successful emergency pain control for posterior rib fractures with ultrasound-guided erector spinae plane block. Am J Emerg Med. 2018;36(8):1391-6. DOI: 10.1016/j.ajem.2017.12.060.

3. Adhikary SD, LiuWM, Fuller E, Cruz-Eng H, Chin KJ. The effect of erector spinae plane block on respiratory and analgesic outcomes in multiple rib fractures: a retrospective cohort study. Anaesthesia. 2019;74(5):585-93. DOI: 10.1111/anae.14579.

4. De Cassai A, Tonetti T. Local anesthetic spread during erector spinae plane block. J Clin Anesth. 2018;48:60-61. DOI: 10.1016/j.jclinane.2018.05.003.

5. Luftig J, Mantuani D, Herring AA, Dixon B, Clattenburg E, Nagdev A. The authors reply to the optimal dose and volume of local anesthetic for erector spinae plane blockade for posterior rib fractures. Am J Emerg Med. 2018;36(6):1103-4. DOI: 10.1016/j.ajem.2018.03.051.

6. Forero M, Rajarathinam M, Adhikary S, Chin KJ. Continuous erector spinae plane block for rescue analgesia in thoracotomy after epidural failure: A case report.
A A Case Rep. 2017;8(10):254-6. DOI: 10.1213/ XAA.0000000000000478.

7. Luis-Moreno JC, Seda-Guzmán M, Luis-Moreno C, LópezRomero JL. Bloqueo del plano del músculo erector de la columna en 4 casos de cirugía torácica videoasistida. Rev Esp Anestesiol Reanim. 2018;65(4):204-8. DOI: 10.1016/j. redar.2017.12.004.

8. Kot P, Rodriguez P, Granell M, Cano B, Rovira L, Morales J, eet al. The erector spinae plane block: a narrative review. Korean J Anesthesiol. 2019;72(3):209-20. DOI: 10.4097/ kja.d.19.00012.

9. Kim M, Moore JE. Chest Trauma: Current Recommendations for Rib Fractures, Pneumothorax, and Other Injuries. Curr Anesthesiol Rep. 2020;10:61-8.

10. Klesius L, Schroeder K. Effective analgesia with bilateral erector spinae plane catheters for a patient with traumatic rib and spine. Curr Anesthesiol Rep. 2019:915-8. DOI: 10.1155/2019/9159878.

11. Hamilton DL, Manickam B. Erector spinae plane block for pain relief in rib fractures. $\mathrm{Br} J$ Anaesth. 2017;118(3):474-5. DOI: 10.1093/bja/aex013.

12. Bermúdez M, Gasalla A, Domínguez E, López González JM, López Carballo C, Pardo F. Erector Spinae Block. A narative review. Centr Eur J Clin Research. 2018;1(1):28-39. DOI: 10.2478/cejcr-2018-0005.

13. Vidal E, Giménez H, Forero M, Fajardo M. Bloqueo del plano del musculo erector espinal: estudio anatómico-cadavérico para determinar su mecanismo de acción. Rev Esp Anestesiol Reanim. 2018;65(9):514-59. DOI: 10.1016/j. redar.2018.07.004.

14. He W, Wu Z, Zu L, Sun H, Yang X. Application of erector spinae plane block guided by ultrasound for postoperative analgesia in breast cancer surgery: A randomized controlled trial. Cancer Commun (Lond). 2020;40(2-3):122-5. DOI: 10.1002/cac2.12013.

15. Malawat A, Verma K, Jethava D, Jethava DD. Erector spinae plane block for complete surgical anaesthesia and postoperative analgesia for breast surgeries: A prospective feasibility study of 30 cases. Indian J Anaesth. 2020;64(2):118-24. DOl: 10.4103/ija.IJA_639_19.

16. Schwartzmann A, Peng P, Maciel MA, Alcarraz P, Gonzalez $X$, Forero $M$. A magnetic resonance imaging study of local anesthetic spread in patients receiving an erector spinae plane block. Can J Anaesth. 2020. DOI: 10.1007/s12630020-01613-8. 